\title{
A Background Timing-Skew Calibration Technique for Time-Interleaved Analog-to-Digital Converters
}

\author{
Chung-Yi Wang, Student Member, IEEE, and Jieh-Tsorng Wu, Member, IEEE
}

\begin{abstract}
This paper presents a background timing-skew calibration technique for time-interleaved analog-to-digital converters (ADCs). The timing skew between any two adjacent analog-digital (A/D) channels is detected by counting the number of zero crossings of the ADCs input while randomly alternating their sampling sequence. Digitally controlled delay units are adjusted to minimize the timing skews among the A/D channels caused by the mismatches among the clock routes. The calibration behaviors, including converging speed and timing jitter, are theoretically analyzed and verified with simulations. A 6-bit 16-channel ADC is used as an example.
\end{abstract}

Index Terms-Analog-digital (A/D) conversion, calibration, timing.

\section{INTRODUCTION}

A TIME-INTERLEAVED analog-to-digital converter (ADC) employs multiple analog-digital (A/D) conversion channels to increase the achievable sampling rate for a given IC technology. Fig. 1 shows a time-interleaved $\mathrm{ADC}$ consisting of $M \mathrm{~A} / \mathrm{D}$ channels, i.e., $\mathrm{ADC}_{1}, \mathrm{ADC}_{2}, \ldots, \mathrm{ADC}_{M}$. Operating at a clock rate of $f_{c}$, each $\mathrm{A} / \mathrm{D}$ channel includes a sample-and-hold amplifier (SHA) for input sampling followed by a quantizer (QTZ) for amplitude digitization. Controlled by clocks with an identical frequency of $f_{c}$ and $M$ uniformly spaced phases $\phi_{1}, \phi_{2}, \ldots, \phi_{M}$, the $M$ SHAs sample the input, $x(t)$, sequentially and periodically. The final digital output code $x[l]$ is produced by multiplexing the outputs from the A/D channels, $x_{1}[k], x_{2}[k], \ldots, x_{M}[k]$. Thus, with each A/D channel operating at a clock rate of $f_{c}$, the overall system's sampling rate becomes $f_{s}=M \times f_{c}$.

Although each A/D channel needs only to operate at the $f_{c}$ clock rate, mismatched A/D characteristics among the channels can degrade the overall A/D resolution. Those mismatches include A/D offset error, A/D gain error, and sampling timing skew. Since those mismatches are sensitive only to temperature and supply voltage variations and vary slowly in time, there are techniques that can calibrate the mismatches in the background without interrupting the normal A/D operation [1], [2].

Consider only the timing-skew issue. If the input is a narrow-band signal, the skew information can be extracted directly from the digital outputs of the A/D channels [1], [2].

Manuscript received January 17, 2005. revised June 1, 2005 and September 4,2005 . This work was supported by the National Science Council of Taiwan, R.O.C., under Contract NSC-93-2220-E-009-005, and by the MediaTek Research Center at National Chiao-Tung University. This paper was recommended by Associate Editor F. Maloberti.

The authors are with the Department of Electronics Engineering, National Chiao-Tung University, Hsin-Chu, Taiwan, R.O.C. (e-mail: jtwu@mail.nctu.edu.tw).

Digital Object Identifier 10.1109/TCSII.2005.861887

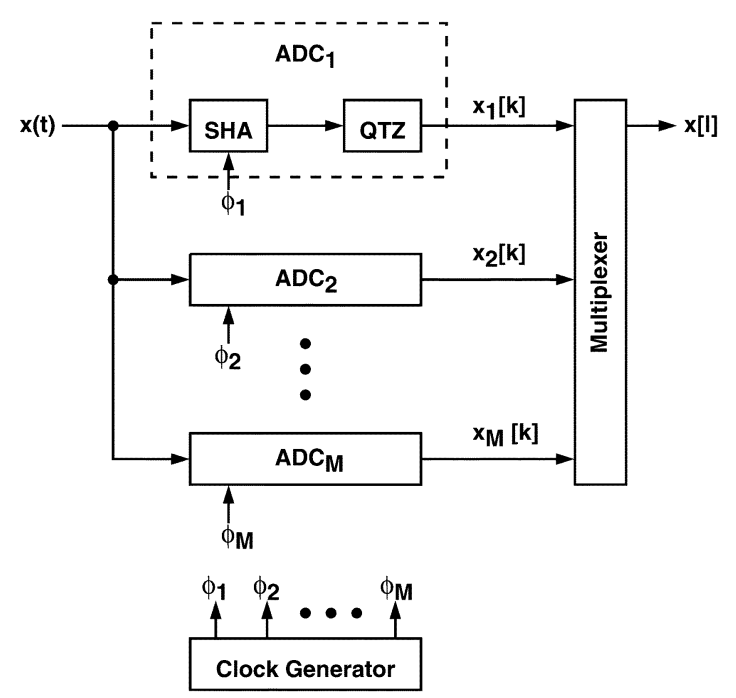

Fig. 1. Time-interleaved ADC architecture.

But these techniques cannot operate with a wide-band input that causes aliasing in each A/D channel. Using a single SHA can avoid the timing skew problem all together. But the SHA has to operate at a clock rate of $M \times f_{c}$. The timing skew can be calibrated by applying a reference signal to the ADC's input and then observing the phase difference between the outputs of adjacent A/D channels [3]. However, the calibration procedures cannot be performed without interrupting the normal A/D operation. It is possible to apply the reference signal for calibration during the normal A/D operation, and then separate the reference signal from the normal input by using correlation technique [4]. In this approach, the injected reference needs to be accurate and it also degrades the available dynamic range of the analog signal path.

This paper presents a background calibration scheme to correct the timing skew. It assumes that the $M$ clocks from the local clock generator $\phi_{1}, \phi_{2}, \ldots, \phi_{M}$ are accurate and have uniformly spaced phases. The mismatches among the clock routes from the clock genertor to the SHAs cause the timing skews. The proposed scheme detects the timing skews by simply counting the number of zero crossings of input signal among the A/D channels while randomly changing their sampling sequence. The obtained informaton is used to adjust the digitally controlled delay units inserted between the clock generator and the SHAs, so that the timing skews can be minimized. The propoed technique allows inputs with wide bandwidth that can cause aliasing in each A/D channel. The maximum allowable bandwith for the input can be as high as $f_{s} / 2$. 
The rest of this paper is organized as follows. Section II introduces the principle of timing-skew detection. Section III describes the proposed calibration scheme between two A/D channels. Section IV describes the calibration scheme for a multi-channel ADC, which is based on the two-channel calibration scheme. Section V presents a 6-bit 16-channel ADC example. Finally, Section VI draws conclusions.

\section{TIMING-SKEW DETECTION}

Assuming all A/D channels shown in Fig. 1 are linear and without gain and offset errors, the digital output of the $\mathrm{ADC}_{j}$ can be expressed as

$$
x_{j}[k]=x\left((M \times k+j) T_{s}+t_{0}+\tau_{j}\right)
$$

where $T_{s}=1 / f_{s}$ is the nominal sampling interval. The $t_{0}$ represents the initial sampling time at $k=0$ for the $\mathrm{ADC}_{1}$. The $t_{0}$ has a value between 0 and $M \times T_{s}$. The $\tau_{j}$ is the timing difference between the clock generator and the $j$ th SHA caused by routing. The $t_{0}$ is defined in such a way that the mean of $\tau_{j}$, for $j=1,2, \ldots M$, is zero, i.e., $\tau_{1}+\tau_{2}+\cdots+\tau_{M}=0$. A timing skew occurs when $\tau_{a} \neq \tau_{b}$ for $a \neq b$. Notable, the sampling interval for each $\mathrm{A} / \mathrm{D}$ channel is $T_{c}=M \times T_{s}$, and the clock frequency is $f_{c}=1 / T_{c}$. Equation (1) neglects the effects of amplitude quantization.

First assume that the sampling rate, $f_{s}$, is larger than the Nyquist sampling frequency of the $x(t)$ input, i.e., larger than twice the $x(t)$ 's bandwidth. Since $x(t)$ is continuous in time and in amplitude, there is one and only one moment between two consecutive sampling instant that the $x(t)$ crosses over the zero if the input's values sampled by the corresponding $\mathrm{A} / \mathrm{D}$ channels, $\mathrm{ADC}_{j}$ and the subsequent $\mathrm{ADC}_{j+1}$, have opposite signs, i.e., $x_{j}[k] \times x_{j+1}[k]<0$. Second, assume that the $x(t)$ is a stationary Gaussian process with zero mean. Then, the probability of a zero crossing between $\mathrm{ADC}_{j}$ and $\mathrm{ADC}_{j+1}, P_{j, j+1}^{z}$, is a bivariate normal distribution [5], [6], and can be expressed as

$$
P_{j, j+1}^{z}=\frac{1}{2}-\frac{1}{\pi} \sin ^{-1} \rho_{j, j+1}
$$

with

$$
\rho_{j, j+1}=\frac{E\left[x_{j} \times x_{j+1}\right]}{\sigma_{j} \times \sigma_{j+1}}
$$

where $\sigma_{j}$ and $\sigma_{j+1}$ are the standard deviations of the $x_{j}$ and $x_{j+1}$ random variables respectively. The $\rho_{j, j+1}$ of (3) denotes the cross-correlation between $x_{j}$ and $x_{j+1}$.

The upper half of Fig. 2 illustrates the proposed timing-skew detection scheme. Two choppers, a clock chopper and a data chopper, are placed at the outputs of clock generator and at the outputs of the A/D channels. The two choppers are controlled by a binary-valued random sequence, $q[k] \in\{-1,+1\}$. When $q[k]=+1$, the choppers' outputs are the same as its corresponding inputs. When $q[k]=-1$, the choppers' outputs are exchanged. Thus, the sampling interval between the $\mathrm{ADC}_{j}$ and the $\mathrm{ADC}_{j+1}$ can be expressed as

$$
\Delta T_{j, j+1}=T_{s}+q[k] \times\left(\tau_{j}-\tau_{j+1}\right) .
$$

As manifested by (7) and (8) shown later, the $P_{j, j+1}^{z}$ is a monotonic function of $\Delta T_{j, j+1}$ for an $x(t)$ input with limited bandwidth. Thus, the polarity of the timing skew $\tau_{j}-\tau_{j+1}$ can

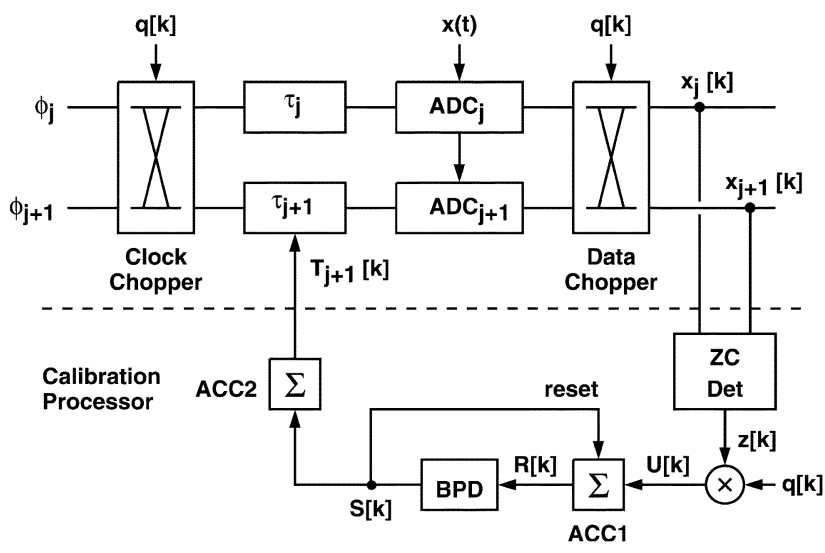

Fig. 2. Timing-skew detection and calibration for two channels.

be detected by observing the change in $P_{j, j+1}^{z}$ whenever $q[k]$ changes. The $q[k]$ is chosen to be random to minimize the input dependence of the detection scheme. To ensure the detection accuracy, it is critical that the clock chopper in Fig. 2 does not introduce additional timing skew.

\section{Two-Channel Timing-Skew CALIBRation}

The bottom half of Fig. 2 shows the block diagram of the proposed timing-skew calibration processor $(\mathrm{CP})$ between the two adjacent $\mathrm{A} / \mathrm{D}$ channels $\mathrm{ADC}_{j}$ and $\mathrm{ADC}_{j+1}$. Since only the polarity of the $\tau_{j}-\tau_{j+1}$ timing skew can be detected, this $\mathrm{CP}$ empolys a similiar approach used in a comparator offset calibration scheme [7]. For the zero-crossing detector (ZC Det), its output $z[k]=1$ whenever $x_{j}[k] \times x_{j+1}[k]<0$, otherwise $z[k]=0$. The $z[k]$ sequence is then correlated with the $q[k]$ sequence and integrated on the $\mathrm{ACC} 1$ accumulator. The ACC1s output is $R[k]$. The rate of long-term change in $R[k]$ is proportional to the probability difference, $\Delta P_{j, j+1}^{z}$

$$
\Delta P_{j, j+1}^{z}=\left.P_{j, j+1}^{z}\right|_{q[k]=+1}-\left.P_{j, j+1}^{z}\right|_{q[k]=-1} .
$$

The bilateral peak detector (BPD) monitors the value of $R[k]$ and generates a corresponding triple-valued output $S[k] \in\{+1,0,-1\}$. The BPD has two thresholds $+N_{C}$ and $-N_{C}$. When $R[k]>+N_{C}, S[k]=+1$. When $R[k]<-N_{C}$, $S[k]=-1$. Otherwise, $S[k]=0$. In addition, the ACC1 accumulator is reset to zero whenever $S[k]=+1$ or $S[k]=-1$. Thus, $-\left(N_{C}+1\right) \leq R[k] \leq+\left(N_{C}+1\right)$, and $S[k]$ can only remain as +1 or -1 for one clock cycle. The $S[k]$ sequence is integrated by the ACC2 accumulator. Its output, $T_{j+1}[k]$, controls the digitally controlled $\tau_{j+1}$ delay unit, such that

$$
\tau_{j+1}[k]=\tau_{j+1,0}+\mu_{t} \times T_{j+1}[k]
$$

where $\mu_{t}$ is the delay unit's step size for digital control and $\tau_{j+1,0}$ is the time delay of $\tau_{j+1}$ when $T_{j+1}[k]=0$. The CP adjusts $\tau_{j+1}$ automatically to minimize the difference between $\tau_{j}$ and $\tau_{j+1}$.

There are two design parameters in this calibration scheme, $\mu_{t}$ and $N_{C}$. Together with $P_{j, j+1}^{z}$ and $\Delta P_{j, j+1}^{z}$, they affect the calibration behaviors, such as the converging speed and the sampling jitter due to the disturbance of the $x(t)$ input. Detailed analyses have been given in [7]. Generally, large $\mu_{t}$ and small $N_{C}$ result in fast converging speed but large timing jitter in $\tau_{j+1}$. 
On the other hand, small $\mu_{t}$ and large $N_{C}$ result in small timing jitter but also slow converging speed.

The calibration behaviors strongly depend on the property of the $x(t)$ input. For a generic $x(t)$ input, the cross-correlation of (3) between two periodic sampling sequences, $x_{a}[k]=$ $x\left(k t_{c}+t_{0}\right)$ and $x_{b}[k]=x\left(k t_{c}+t_{s}+t_{0}\right)$, can be expressed as $\rho\left(t_{0}, t_{s}, t_{c}\right)$. The $t_{c}$ is the sampling interval for each of the sampling sequence, the $t_{s}$ is the sampling time difference between the two sequences, and the $t_{0}$ is the initial sampling time for $x_{a}[0]$. Notably, the $\rho\left(t_{0}, t_{s}, t_{c}\right)$ is a periodic function of $t_{0}$ with a period of $t_{c}$. In the case of a time-interleaved ADC, we also have $t_{s} \leq t_{c} / 2$. From (2), the corresponding zero-crossing probability between the two sampling sequences can be expressed as $P^{z}\left(t_{0}, t_{s}, t_{c}\right)$. Analogous to the probability density function, the zero-crossing density, defined as the zero-crossing probability per unit $t_{s}$ time, can be expressed as

$$
\begin{aligned}
Z_{R}\left(t_{0}, t_{c}\right) & \equiv \lim _{t_{s} \rightarrow 0} \frac{P^{z}\left(t_{0}, t_{s}, t_{c}\right)}{t_{s}} \\
& =\frac{1}{\pi} \times\left.\left[-\frac{\partial^{2} \rho\left(t_{0}, t_{s}, t_{c}\right)}{\partial t_{s}^{2}}\right]^{1 / 2}\right|_{t_{s}=0} .
\end{aligned}
$$

For a $M$-channel time-interleaved ADC with $T_{s}$ sampling interval between the adjacent channels, the single-channel sampling interval is $T_{c}=M \times T_{s}$. Assume the timing skew between the $\mathrm{ADC}_{j}$ and the $\mathrm{ADC}_{j+1}$ is small, i.e., $\Delta \tau_{j} \equiv \tau_{j}-\tau_{j+1} \ll$ $T_{s}$, the probability difference, $\Delta P_{j, j+1}^{z}$, can then be approximated by

$$
\Delta P_{j, j+1}^{z}=\left[Z_{R}\left(t_{0}, T_{c}\right)+Z_{R}\left(t_{0}+T_{s}, T_{c}\right)\right] \times \Delta \tau_{j} .
$$

From (7), it can be shown that $Z_{R}\left(t_{0}, t_{c}\right) \geq 0$. Thus, $\Delta P_{j, j+1}^{z}$ has the same polarity as $\Delta \tau_{j}$. Furthermore, the zero-crossing probability can be expressed as

$$
P_{j, j+1}^{z}=\int_{t_{0}}^{t_{0}+T_{s}} Z_{R}\left(t, T_{c}\right) d t
$$

Both $P_{j, j+1}^{z}$ and $\Delta P_{j, j+1}^{z}$ are required in estimating the converging speed and timing jitter of the calibration process [7].

Consider the the system shown in Fig. 2. If $\Delta P_{j, j+1}^{z} / \Delta \tau_{j}=$ $Z_{R}\left(t_{0}, T_{c}\right)+Z_{R}\left(t_{0}+T_{s}, T_{c}\right)$ is a constant, then the system's transient behavior can be modeled as a single-pole system with a time constant expressed as [7]

$$
\tau_{c}=\frac{N_{C}}{\mu_{t}} \times \frac{2}{Z_{R}\left(t_{0}, T_{c}\right)+Z_{R}\left(t_{0}+T_{s}, T_{c}\right)} .
$$

As an example, let $x(t)$ be a single-tone sine wave, i.e., $x(t)=A \sin \left(2 \pi f_{i} t\right)$, which has a frequency of $f_{i}$ and a constant amplitude of $A$. Its corresponding $\rho\left(t_{0}, t_{s}, t_{c}\right)$ is $\cos \left(2 \pi f_{i} t_{s}\right)$, and the corresponding $Z_{R}\left(t_{0}, t_{c}\right)$ can be expressed as

$$
Z_{R}\left(t_{0}, t_{c}\right)= \begin{cases}2 f_{i}, & \frac{f_{i}}{f_{c}} \neq \frac{a}{b} \\ \sum_{n=0}^{a-1} \frac{2}{b} \delta\left(t_{0}-n \cdot \frac{t_{c}}{a}\right), & \frac{f_{i}}{f_{c}}=\frac{a}{b} b \text { is even } \\ \sum_{n=0}^{2 a-1} \frac{1}{b} \delta\left(t_{0}-n \cdot \frac{t_{c}}{2 a}\right), & \frac{f_{i}}{f_{c}}=\frac{a}{b} b \text { is odd }\end{cases}
$$

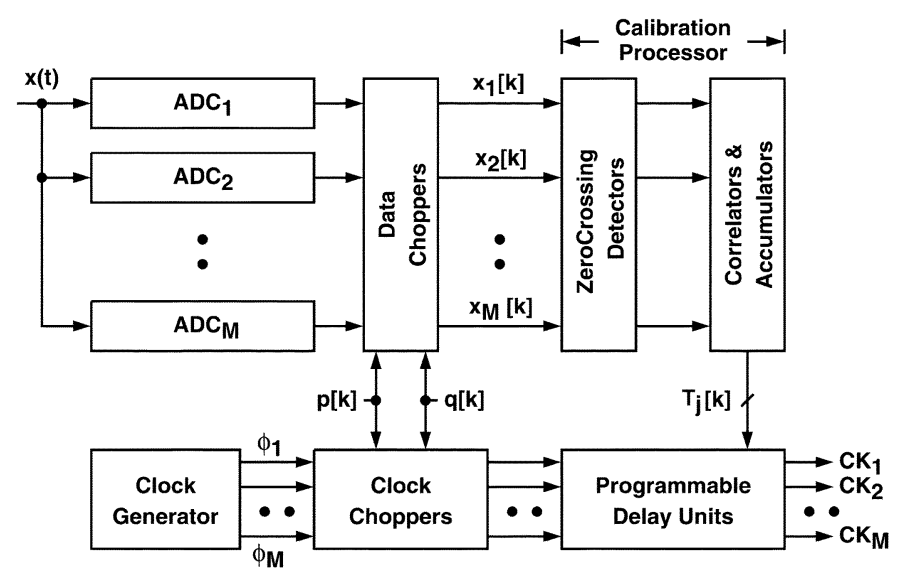

Fig. 3. Full-system timing-skew calibration.

where $f_{c}=1 / T_{c}$ is the sampling rate for a single channel, and $a$ and $b$ are two mutually prime positive integers. If the $f_{i} / f_{c}$ ratio is irrational, i.e., $f_{i} / f_{c} \neq a / b$, the zero-crossing density, $Z_{R}$, is equal to $2 f_{i}$, and independent of $t_{0}$ and $t_{c}$. If $f_{i} / f_{c}=a / b$, the input sine wave synchronizes with the $f_{c}$ sampling clock. Thus, within any time period of the $f_{c}$ clock, there are only a finite number of instants at which the zero-crossings can occur. If $b$ is even, there are $a$ possible uniformly spaced zero-crossing instants. On the other hand, if $b$ is odd, there are $2 a$ possible uniformly spaced zero-crossing instants. The proposed timing-skew calibration scheme cannot function properly with a synchronous input, unless the coressponding $a$ is sufficiently large so that the time interval between the zero-crossings is smaller than the required timing resolution.

\section{Multi-Channel Timing-Skew Calibration}

Fig. 3 shows the calibration scheme for the entire $M$-channel time-interleaved ADC. The clock generator produces $M$ clocks with an identical frequency of $f_{c}$ and equally spaced phases. The clocks pass through the clock choppers and the digitally controlled delay units to generate $\mathrm{CK}_{1}, \mathrm{CK}_{2}, \ldots, \mathrm{CK}_{M}$ which control the sampling timing of $\mathrm{ADC}_{1}, \mathrm{ADC}_{2}, \ldots, \mathrm{ADC}_{M}$ repectively. The calibration processor $(\mathrm{CP})$ adjusts the digitally controlled delay units to minimize the timing skews among the A/D channels. The timing skews are caused by mismatches among the clock routes from the outputs of clock choppers to the sample-to-hold amplifiers in the A/D channels. The CP is pure digital and operate at a clock rate of $f_{c}$. It consists of only comparators, adders and registers, and requires no multi-bit multiplier.

A pairing scheme is proposed so that 1) the two-channel timing-skew calibration can be executed simultaneously on the selected pairs of A/D channels; 2) each clock chopper only swaps the sampling clocks of adjacent A/D channels; and 3) timing skews of all $A / D$ channels are minimized relative to a single reference channel. As shown in Fig. 3, there are two independent random sequences, $p[k] \in\{-1,+1\}$ and $q[k] \in\{-1,+1\}$ for the control of the clock choppers and the data choppers. Fig. 4 illustrates the proposed pairing scheme 


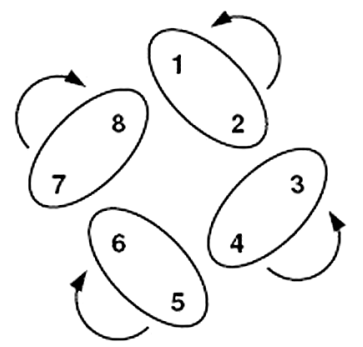

$p[k]=1$

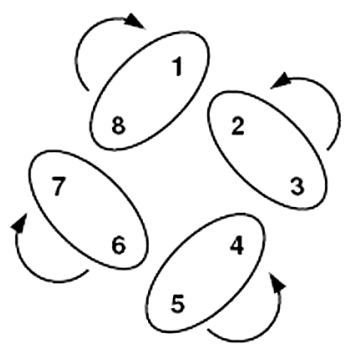

$p[k]=0$
Fig. 4. Proposed pairing scheme for an 8-channel system.

for an 8-channel time-interleaved ADC. When $p[k]=+1$, the following calibration pairs are selected for simultaneous calibration: $(1,2),(3,4),(8,7),(6,5)$. For each calibration pair, $(a, b)$, the $q[k]$ sequence toggles the corresponding choppers to altenerate the sampling sequence of $\mathrm{ADC}_{a}$ and $\mathrm{ADC}_{b}$. The $\mathrm{CP}$ then adjusts the $b$ th delay unit to minimized the timing skew between the two channels. When $p[k]=-1$, the following calibration pairs are selected for simultaneous calibration: (2, $3),(4,5),(1,8),(7,6)$. This pairing scheme assigns $\mathrm{ADC}_{1}$ as the reference channel. For other A/D channel, its corresponding delay unit is ajusted so that its timing skew with the reference channel is eventually minimized.

In the above pairing scheme, the sampling interval of each individual $\mathrm{A} / \mathrm{D}$ channel is no longer a constant $M T_{s}$, due to the reordering of the sampling sequence. The two random sequences, $p[k]$ and $q[k]$, are operated at the $f_{c}$ clock, and they can change state only after the present state has been applied to all $\mathrm{A} / \mathrm{D}$ channels. If $p[k]$ is restricted to change only during $q[k]=+1$, the sampling interval for each A/D channel can be confined to vary between $(M-1) T_{s}$ and $(M+1) T_{s}$.

Consider only the timing jitter caused by the calibration process using the pairing scheme just described. Let $\mathrm{ADC}_{1}$ be the reference channel in a $M$-channel ADC, and all calibration pairs employ identical $\mu_{t}$ and $N_{C}$ parameters. Since $\mathrm{ADC}_{1}$ is the reference channel, the corresponding $\tau_{1}$ is not adjusted, thus its jitter standard deviation $\sigma\left(\tau_{1}\right)=0$. For $\mathrm{ADC}_{2}$, the corresponding $\tau_{2}$ is adjusted toward $\tau_{1}$, resulting in a jitter standard deviation of $\sigma\left(\tau_{2}\right)$. For $\mathrm{ADC}_{3}$, the corresponding $\tau_{3}$ is adjusted toward $\tau_{2}$, resulting in a jitter standard deviation of $\sqrt{2} \sigma\left(\tau_{2}\right)$. In general, for $\mathrm{ADC}_{j+1}$ where $j \geq 2$, the corresponding $\tau_{j+1}$ is adjusted toward $\tau_{j}$, and its jitter standard deviation can be expressed as

$$
\sigma\left(\tau_{j+1}\right)=\sqrt{j} \times \sigma\left(\tau_{2}\right) .
$$

On the other side of $\mathrm{ADC}_{1}, \mathrm{ADC}_{M}$ has the same jitter standard deviation as $\mathrm{ADC}_{2}, \mathrm{ADC}_{M-1}$ has the same jitter standard deviation as $\mathrm{ADC}_{3}$, and etc. In the 8-channel example, the $\mathrm{ADC}_{5}$ has largest timing jitter. To reduce $\sigma\left(\tau_{j+1}\right)$, the CP can use larger $N_{C}$ value when calibrating the timing skew of the $\mathrm{ADC}_{j+1}$.

In the proposed pairing sheme for multichannel calibration, the use of the $p$ random sequence increases the response time of

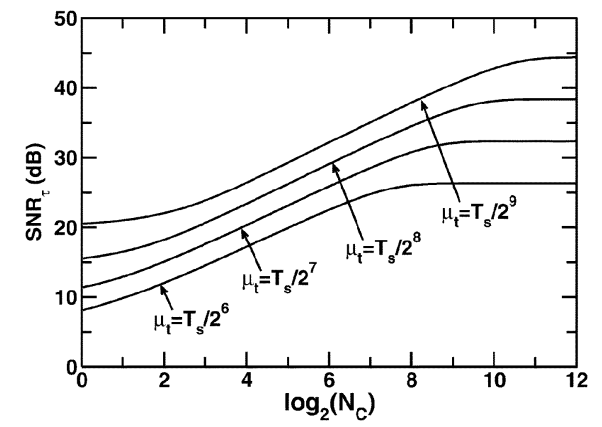

Fig. 5. $\mathrm{SNR}_{\tau}$ versus $N_{C}$ and $\mu_{t}$ for the 6-bit 16-channel ADC example.

the system by a factor of two. If $\Delta P_{j, j+1}^{z} / \Delta \tau_{j}=Z_{R}\left(t_{0}, T_{c}\right)+$ $Z_{R}\left(t_{0}+T_{s}, T_{c}\right)$ is a constant, then the system's transient behavior can also be modeled as a single-pole system with a time constant expressed as

$$
\tau_{s}=2 \tau_{c}
$$

where $\tau_{c}$ is defined in (10).

It is imperative to carefully choose the timing of $p[k]$ and $q[k]$ for controlling the clock choppers, so that undesirable glitches are not generated in the clocks when the choppers are toggled.

\section{6-Bit 16-ChANNEl Design ExAmPle}

A 6-bit 16-channel ADC, i.e., $M=16$, is used as a design example. The sampling interval between the adjacent channels is $T_{s}$, and the sampling period for each channel is $T_{c}=$ $M \times T_{s}=16 T_{s}$. The single-channel sampling rate is $f_{c}=1 / T_{c}$ and the effective sampling rate for the entire $\mathrm{ADC}$ is $f_{s}=1 / T_{s}$. The ADC is similiar to the system illustrated in Fig. 4 and employs the pairing scheme described in Section IV. Identical $\mu_{t}$ and $N_{C}$ parameters are used in all calibration procedures. The input is assumed to be an asynchronous sine wave, i.e., $x(t)=$ $A \sin \left(2 \pi f_{i} t\right)$ and the $f_{i} / f_{c}$ ratio is irrational. From (11), the corresponding zero-crossing density is $Z_{R}=2 f_{i}$.

Consider the timing jitter caused by the CP. Besides $\mu_{t}$ and $N_{C}$, the jitter standard deviations depend only on the $\Delta P_{j, j+1}^{z} / P_{j, j+1}^{z}$ ratio [7]; thus, it is not a function of the $f_{i}$ input frequency for the single-tone input case. The $\mathrm{ADC}_{1}$ is assigned as the reference channel, thus $\sigma\left(\tau_{1}\right)=0$. Both the $\mathrm{ADC}_{2}$ and $\mathrm{ADC}_{16}$ have the same jitter standard deviation, i.e., $\sigma\left(\tau_{2}\right)=\sigma\left(\tau_{16}\right)$. The value of $\sigma\left(\tau_{2}\right)$ can be calculated from $\mu_{t}$ and $N_{C}$ [7]. For other A/D channels, their corresponding jitter standard deviation can be calculated using (12). The $\mathrm{ADC}_{9}$ is the most remote $\mathrm{A} / \mathrm{D}$ channel away from the $\mathrm{ADC}_{1}$; it has the worst-case jitter of $\sigma\left(\tau_{9}\right)=\sqrt{8} \times \sigma\left(\tau_{2}\right)$.

Assume the ADC's output contains only noises caused by sampling timing skew. Then, the output's signal-to-noise ratio can be expressed as

$$
\mathrm{SNR}_{\tau}=\frac{1}{4 \pi^{2} f_{i}^{2} \sigma^{2}(\tau)}
$$

where $\sigma^{2}(\tau)$ is the timing-skew standard deviation. Fig. 5 shows the calculated $\mathrm{SNR}_{\tau}$ against different values of $N_{C}$ and $\mu_{t}$ for the 16-channel ADC example. The input frequency is assumed 


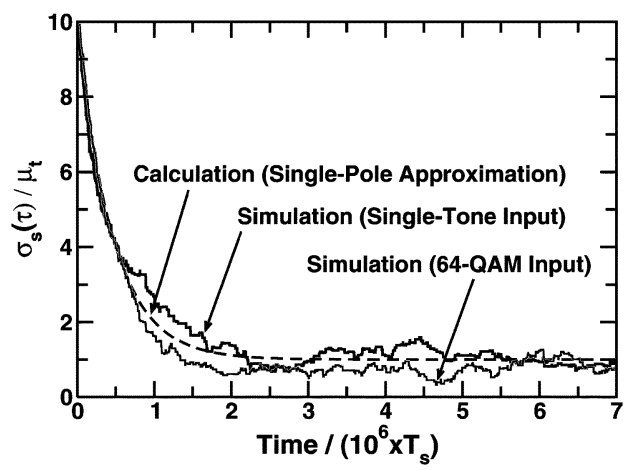

Fig. 6. Timing-skew settling behavior for the 6-bit 16-channel ADC example.

to be close to the Nyquist frequency, i.e., $f_{i} \approx f_{s} / 2$. For this ADC example, the $\sigma^{2}(\tau)$ in (14) can be expressed as

$$
\sigma^{2}(\tau)=\sum_{j=1}^{16} \sigma^{2}\left(\tau_{j}\right)=\sigma^{2}\left(\tau_{2}\right) \times \frac{1}{16} \sum_{j=-7}^{8}|j|=4 \sigma^{2}\left(\tau_{2}\right)
$$

As $N_{C}$ increases, $\mathrm{SNR}_{\tau}$ is saturated to a value determined by the $\mu_{t}$. To achieve 6-bit resolution, $N_{C}=2^{9}$ and $\mu_{t}=T_{s} / 2^{8}$ are chosen in this design example, In such case, $\sigma(\tau) \approx \mu_{t}$ and $\mathrm{SNR}_{\tau} \approx 37 \mathrm{~dB}$.

For the case of an asynchronous single-tone input with $Z_{R}=$ $2 f_{i}$, the ADCs transient behavior can be modeled as a singlepole system with a time constant of $\tau_{s}=N_{C} /\left(f_{i} \times \mu_{t}\right)$, which is obtained using (13) and (10). Fig. 6 shows the settling behavior of the timing-skew spatial standard deviation, $\sigma_{s}(\tau)$ for this ADC example. The spatial standard deviation is collected by recording $\tau_{j}$ for $j=1,2, \ldots, 16$ at a given time. Calibration parameters are $N_{C}=2^{9}$ and $\mu_{t}=T_{s} / 2^{8}$. Results from both simulations and calculations using the single-pole model are shown in Fig. 6. For the single-tone simulation case, the input is a sine wave with a frequency of $(2345 / 8192) f_{s} \approx 0.29 f_{s}$. The initial value for $\sigma_{s}(\tau)$ is set to $10 \mu_{t}$. When settled, the steady-state $\sigma_{s}(\tau)$ is close to $1 \mu_{t}$. For the broad-band simulation case, the input is a 64-QAM signal. Its carrier has a frequency of $(2345 / 8192) f_{s}$. The symbols are a random sequence, and the symbol rate is $(1739 / 8192) f_{s}$. The settling behavior of the broad-band case is similar to that of the simulation case with a single-tone input at the carrier frequency.

In the single-tone simulation case, the ADC's output spectrum exhibits visible spurious tones before calibration, and the signal-to-noise-and-distortion ratio (SNDR) is calculated to be $23.1 \mathrm{~dB}$. When the calibration is turned on, all the visible tones are suppressed and the SNDR is improved to $37.0 \mathrm{~dB}$, as predicted in Fig. 5. For the broad-band simulation case, the SNDR of the ADCs output is $22.4 \mathrm{~dB}$ before calibration, and is improved to $36.1 \mathrm{~dB}$ when the calibration is turned on.

\section{EFFECTS OF GAIN/OFFSET Mismatches}

Since only the zero crossings are collected in the the proposed timing-skew calibration scheme, the inter-channel gain mismatches do not affect the calibration behaviors. However, depending on the input condition, the calibration effectiveness may be sensitive to the offset mismatches. Consider two adja- cent $\mathrm{A} / \mathrm{D}$ channels, $\mathrm{ADC}_{j}$ and $\mathrm{ADC}_{j+1}$. If the $\mathrm{ADC}_{j}$ has an input offset of 0 while the $\mathrm{ADC}_{j+1}$ has an input offset of $O_{j+1}$, the zero-crossing probability between the two channels, $P_{j, j+1}^{z}$, is deviated from (2) by an amount of $\delta P_{j, j+1}^{z}$. The $\delta P_{j, j+1}^{z}$ can be approximated by

$$
\delta P_{j, j+1}^{z} \approx \frac{\rho_{j, j+1}}{2 \pi \sqrt{1-\rho_{j, j+1}^{2}}} \times \frac{O_{j+1}^{2}}{E\left[x_{j+1}^{2}\right]} .
$$

If the input exhibits large $\rho_{j, j+1}$ cross-correlation property, the $P_{j, j+1}^{z}$ is more sensitive to the offset mismatch. The offset sensitivity is reduced when large-power input is applied, since largepower input leads to large $E\left[x_{j+1}^{2}\right]$ value.

It is necessary to keep $\delta P_{j, j+1}^{z}$ of (16) much smaller than $\Delta P_{j, j+1}^{z}$ of (5), so that the analyzes of the previous sections can remain valid. There are calibration techniques that can minimize the offset mismatches [1], [8].

\section{CONCLUSION}

The probability of input's zero crossing between two sampling channels is related to the cross-correlation of the sampled data, and can be expressed as a bivariate normal distribution. Theoretical analyzes can be carried out to estimate the converging speed and jitter behavior of the proposed calibration scheme, as well as its sensitivity to the inter-channel offset mismatch. The analyzes are valid even for wide-band input that causes aliasing in each $\mathrm{A} / \mathrm{D}$ channel, as long as the zero-crossing density $Z_{R}\left(t_{0}, t_{c}\right)$ of (7) can be found.

The clock choppers and the digitally controlled delay units are crucial in this calibration scheme. The clock choppers must not cause additional timing skew and unwanted glitches in the clock signals. The delay units must provide the $\mu_{t}$ step size for digital control; and its adjustable range must cover all possible timing skew variations.

\section{REFERENCES}

[1] S. M. Jamal, D. Fu, N. C.-J. Chang, P. J. Hurst, and S. H. Lewis, “A 10-b 120-Msample/s time-interleaved analog-to-digital converter with digital background calibration," IEEE J. Solid-State Circuits, vol. 37, no. 12, pp. 1618-1627, Dec. 2002.

[2] J. Elbornsson, F. Gustafsson, and J.-E. Eklund, "Blind adaptive equalization of mismatch errors in a time-interleaved A/D converter system," IEEE Trans. Circuits Syst. I, Reg. Papers, vol. 51, no. 1, pp. 151-158, Jan. 2004.

[3] K. Poulton, R. Neff, B. Setterberg, B. Wuppermann, T. Kopley, R. Jewett, J. Pernillo, C. Tan, and A. Montijo1, "A 20 GS/s 8 b ADC with a $1 \mathrm{MB}$ memory in $0.18 \mu \mathrm{m}$ CMOS," in Proc. IEEE Int. Solid-State Circuits Conf., Feb. 2003, pp. 318-496.

[4] H. Jin and E. K. F. Lee, "A digital-background calibration technique for minimizing timing-error effects in time-interleaved ADCs," IEEE Trans. Circuits Syst. II, Analog Digit. Signal Process., vol. 47, no. 7, pp. 603-613, Jul. 2000.

[5] H. Stark and J. W. Woods, Probability Random Processes, and Estimation Theory for Engineers, 2nd ed. Englewood Cliffs, NJ: Prentice-Hall, 1995.

[6] J. T. Barnett and B. Kedem, "Zero-crossing rates of functions of Gaussian processes," IEEE Trans. Inf. Theory, vol. 37, no. 7, pp 1188-1194, Jul. 1991.

[7] C.-C. Huang and J.-T. Wu, "A background comparator calibration technique for flash analog-to-digital converters," IEEE Trans. Circuits Syst. I, Reg. Papers, vol. 52, no. 9, pp. 1732-1740, Sep. 2005.

[8] H. van der Ploeg, G. Hoogzaad, H. A. H. Termeer, M. Vertregt, and R. L. J. Roovers, "A 2.5 V 12-b 54-Msample/s 0.25- $\mu \mathrm{m}$ CMOS ADC in 1 - $\mathrm{mm}^{2}$ with mixed-signal chopping and calibration," IEEE J. Solid-State Circuits, vol. 36, no. 12, pp. 1859-1867, Dec. 2001. 